\title{
Investigating the Effect of Display Pixel Density on the Minimal Legible Size of Cartographic Symbols
}

\author{
Florian Ledermann \\ TU Wien, florian.ledermann@tuwien.ac.at
}

Keywords: Map perception, map symbols, user study, cognition, controlled experiment

\begin{abstract}
:
Current cartographic design guidelines generally demand that maps intended for screen presentation use larger symbology than paper-based maps, due to the reduced fidelity of graphical reproduction (Neudeck, 2001; Lobben \& Patton, 2003; Jenny et al., 2008). However, such recommendations were generally derived from the state of technological development of display hardware around the turn of the millennium, when most desktop monitors were limited at pixel densities around 100 pixels per inch (ppi) (Malić, 1998), and the modern smartphone hadn't been invented. In recent years, screens for mobile devices, but also desktop screens, have become available with ever higher pixel densities. Today, there are virtually no technical limitations in manufacturing displays of ultra-high pixel densities, and devices with pixel densities of several thousand pixels per inch have been produced for special purposes such as AR headsets (Katsui et al., 2019). The limit of pixel density for commercially available desktop monitors currently lies at 280ppi (Dell UltraSharp UP3218K), and for mobile phones at 801ppi (Sony Xperia Z5 Premium). However, guidelines for the minimum dimensions of cartographic symbology have not been updated to reflect those developments. Does the dramatically increased fidelity of digital displays observed in recent years mean that cartographers can now revert to the minimum dimensions that have been established for printed maps? Can even smaller symbol sizes than on paper be used for smartphones due to the increased contrast ratio and the reduced viewing distance? Or does the recommendation to use larger symbology for screens still hold? Also, will cartographic depictions benefit from a continued increase in commonly available pixel density, or is there a point at which further increase of fidelity does not result in increased legibility of cartographic symbology due to limitations of the human perceptual system?
\end{abstract}
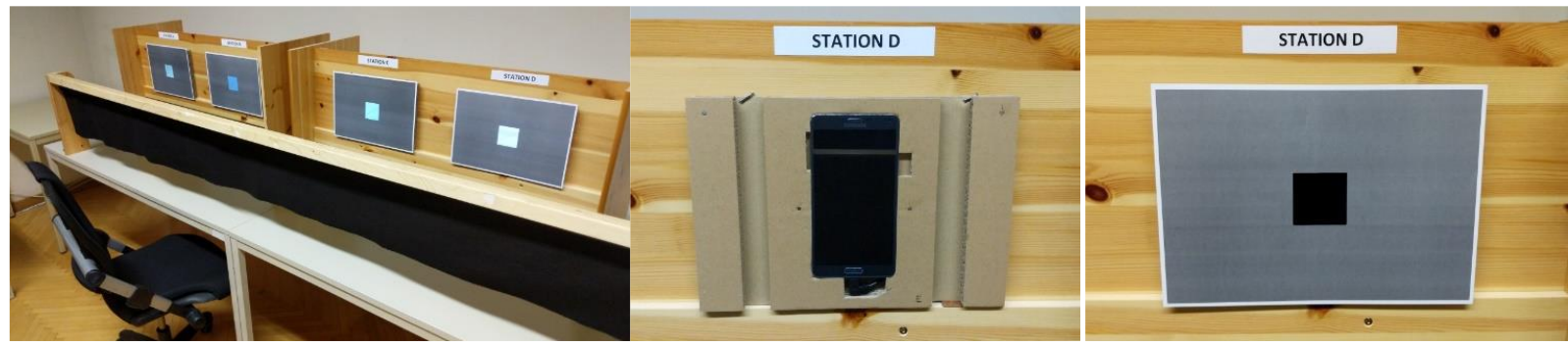

Figure 1. Lab setup for the study. Left: viewing stations with mobile phones of varying pixel densities mounted behind bezels, rail to ensure constant viewing distance, curtain to minimize reflections. Middle: mobile phone mounting fixture. Right: Bezel covering mounting fixture to reveal an area of identical size for each station.

This paper reports early findings of a lab study that attempts to experimentally establish the limits of legibility for fundamental cartographic symbology at various pixel densities on modern smartphone screens. In a controlled setting, mobile phones of varying pixel densities $(228 / 342$ / 522 / 801 ppi, a factor of $\approx \times 1.5$ between each pair) were mounted behind bezels revealing only a square portion of identical size of each phone display. In front of these viewing stations, a rail was mounted to ensure equal viewing distance of approximately $35 \mathrm{~cm}$ for each display (see Fig. 1). Participants were shown a sequence of stimuli on each screen, and had to select the symbol best matching the particular stimulus by pressing the corresponding on-screen button on a separate device (a n-alternative-forced-choice design). Stimulus sizes were adjusted using a "staircase" procedure, in which upon three consecutive correct responses the stimulus size was decreased, and upon one incorrect response the stimulus size was increased. Using this procedure, we could establish the limit at which stimuli could still be reliably distinguished from one another for each participant and display.

The experiment was run with 28 participants recruited among student volunteers. Each participant would perform the tasks of the experiment on all four display stations in randomized order. At each station, an identical sequence of tasks was run, each with a specific set of stimuli to distinguish (see Fig. 2) - (1) a "tumbling E" (randomly rotated in any of 
the 4 cardinal directions), which is established as a test for visual acuity and let us establish the visual acuity of the participant; (2) three or four parallel lines or a grey line, random orientation; (3) dotted, dashed, dot-dash and solid lines, random orientation; (4) point symbols taken from the "Auckland Optotypes" symbol set (Hamm et al., 2018); (5) Point symbols, drawn with a white-black-white outline against a grey background ("vanishing" into grey when beyond legibility) ; (6) Short words (made up to look plausible as a toponym without being a dictionary word or a well-known toponym) with a white outline against grey background, rotated randomly $\pm 90^{\circ}$. The stimuli of tasks $2-6$ have been designed such that conclusions of relevance to cartographic symbology can be made - line symbology (tasks 2,3,5), point symbology (tasks 4,5) and map labels (task 6).

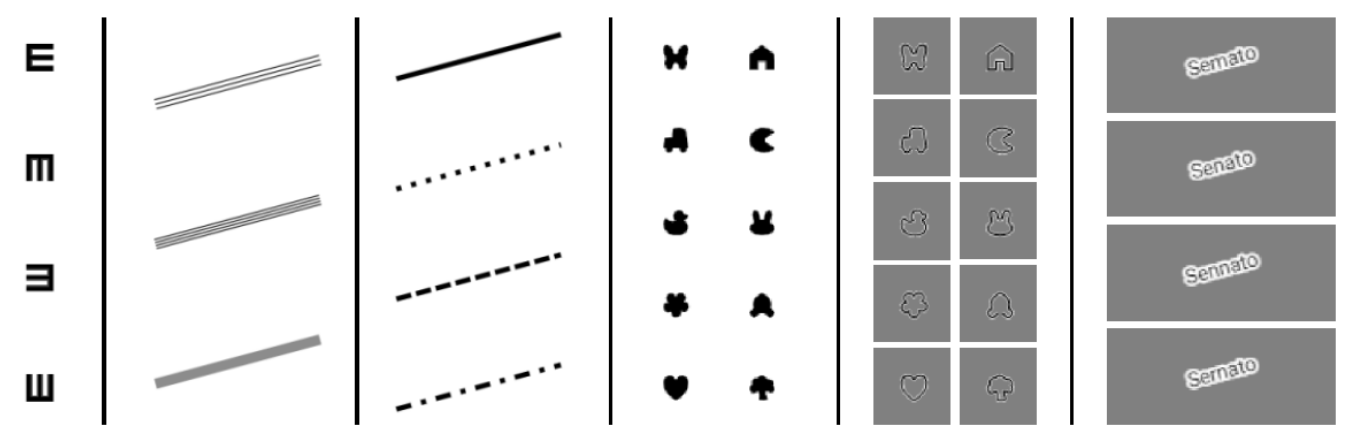

Figure 2. Types of stimuli which participants were asked to distinguish in the experiment. (1) Tumbling E's (2) parallel lines (3) dashed/dotted lines (4) "Auckland Optotypes" symbols (5) "vanishing" symbols (6) word variants. (The orientation of stimuli for tasks 2,3 and 6 was randomized for each trial in the experiment).

In line with our hypotheses, the display with lowest pixel density (228ppi) was outperformed significantly by the one of next higher pixel density (342ppi) in five out of six tasks. The display of yet higher density (522ppi) outperformed the 342ppi display significantly only in two tasks (parallel lines (2) and vanishing symbols (5)), while, surprisingly, performing significantly worse in the tumbling E task (1). The display with highest pixel density (801ppi) outperformed the 522ppi display significantly only in the tumbling E tasks (with no significant improvement over the 342ppi display in that task), making the display of highest pixel density the only display on which participants did not perform significantly worse in any task than on any other display. However, average performance on this display was worse (but not strongly significant) than on the 522ppi display in two tasks (parallel lines and vanishing symbols). Contrary to our hypotheses, for the text recognition task (6) no display performed significantly better than any other display (see fig. 3 for plots of participants' thresholds for a selection of tasks).
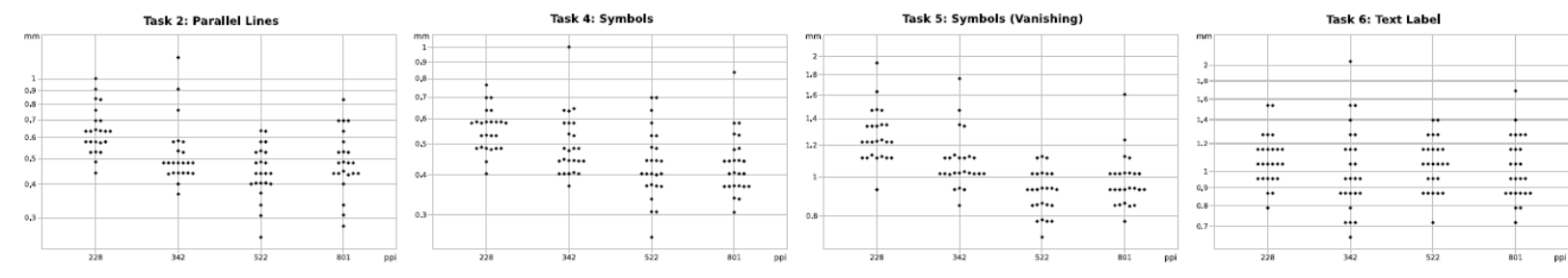

Figure 3. Beeswarm plots of participants' thresholds for correctly identified stimuli. Stimulus size is given on the y-axis (log scale, note the different levels between tasks), with smaller values considered better performance. Pixel density of the display is shown as an ordinal variable on the $\mathrm{x}$-axis.

In the paper, we give a detailed report and statistical analysis of the results of the experiment and attempt to derive updated guidelines for minimum cartographic symbol sizes, both for the general case in which the target display device is unknown to the map producer (e.g. for online maps or apps) as well as for settings in which a high-density display device can be reliably provided (for example for fixed installations or in organizations in which specific devices could be deployed, e.g. emergency services). We will furthermore present initial ideas for a sampling-theoretical model that explains some of the more surprising results of the study, particularly the poor performance of the 522ppi display in the "tumbling E" tasks and the practically equal level of performance across all four displays for the text recognition task. 